\title{
Intravenous lipid emulsion - rescued at LAST
}

\author{
S. J. Ciechanowicz ${ }^{1}$ and V. K. Patil ${ }^{2}$
}

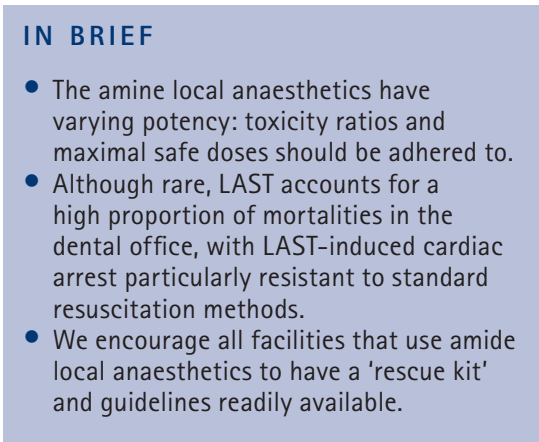

The accidental overdose of local anaesthetics may prove fatal. The commonly used amide local anaesthetics have varying adverse effects on the myocardium and beyond a certain dose all are capable of causing death. Local anaesthetics are the most frequently used drugs in dentistry and although uncommon, local anaesthetic systemic toxicity (LAST) accounts for a high proportion of mortalities in the dental office, with local anaesthetic-induced cardiac arrest particularly resistant to standard resuscitation methods. Over the last decade there has been convincing evidence of using intravenous lipid emulsions as a rescue in local anaesthetic - cardiotoxicity and anaesthetic organisations over the globe have developed guidelines on the use of this drug. Despite this, however, awareness among practitioners appears to be lacking. All who use local anaesthetics in their practice should have an appreciation of patients at high risk of toxicity, early symptoms and signs of toxicity, preventative measures when using these drugs and the initial management of systemic toxicity with intravenous lipid emulsion. In this review we intend to discuss the pharmacology and pathophysiology of local anaesthetic toxicity, and the rationale for intravenous lipid emulsion therapy.

Local anaesthetics (LAs) can be defined as drugs that reversibly block transmission of a nerve impulse, without affecting consciousness. Koller first used cocaine successfully in ophthalmic surgery as a topical anaesthetic. Halsted and Hall took more invasive steps by directly injecting cocaine into oral cavity nerves in order to produce anaesthesia for removal of a wisdom tooth. ${ }^{1}$ Over the years the relatively safer amino amides came into use with lignocaine appearing in 1948, now the most commonly used LA in dentistry in the US.

Amino amides mepivacaine, prilocaine and bupivacaine were all developed by 1963 and all have roles in modern dentistry. In 1969, articaine was synthesised by chemist Muschaweck, and with its potency and safety profile, is now the

\footnotetext{
"Queen's Hospital, Anaesthetics, Rom Valley Way, Romford, Essex, RM7 OAG; ${ }^{2}$ Oueen's Hospital, Anaesthetics, Rom Valley Way, Romford, Essex, RM7 $O A G{ }^{*}$ Correspondence to: Dr Sarah Ciechanowicz Email: sciechanowicz@doctors.org.uk; Tel: 07729104960

Refereed Paper

Accepted 8 December 2011

DOI: $10.1038 /$ sj.bdj.2012.187

${ }^{\circledR}$ British Dental Journal 2012; 212: 237-241
}

most common LA for dental procedures in most of Europe. ${ }^{2}$ Despite these efforts, all of the amide LAs harbour varying levels of cardiovascular (CVS) and central nervous system (CNS) toxicity that is still a major complication seen today.

\section{MECHANISM OF ACTION}

The physicochemical properties of LAs determine their properties as anaesthetic agents. They have three structural groups: an aromatic ring, connecting group (ester or amide) and an ionisable amino group. These lipid-soluble hydrophobic aromatic groups and charged hydrophilic amide groups enable them to exert their effects by two mechanisms: in their uncharged (un-ionised) state they are lipid soluble and able to traverse the lipid bilayer of the neuronal cell membrane, to then gain a hydrogen ion and become ionised making them able to bind intracellularly to voltage-gated sodium channels, rendering the channel reversibly inactive, and so unable to allow for sodium entry to generate and propagate the action potential $^{3}$ (Fig. 1). Binding can also occur to the closed sodium channel to retain its inactive state. Secondly, LAs have direct effects

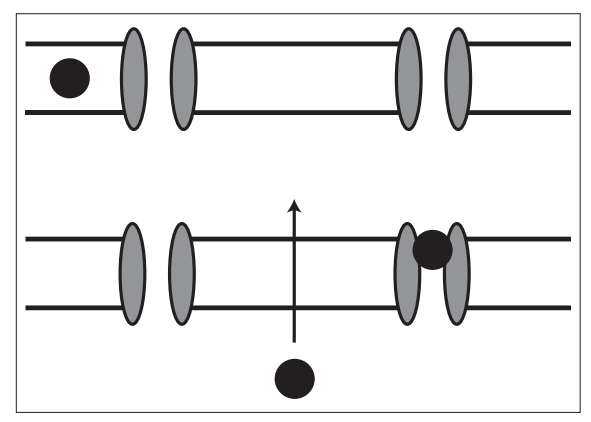

Fig. 1 Mechanism of action of local anaesthetics. Un-ionised LA enters nerve axon and becomes ionised to block sodium channels. LA also has direct effects by expanding the cell membrane to increase fluidity

on the lipid bilayer, disrupting impulses by incorporating into the cell membrane, causing expansion. ${ }^{4,5}$ The sensitivity of nerve fibres depends upon their axonal diameter and degree of myelination with small, more susceptible myelinated fibres. Generally the small pain and temperature fibres (C unmyelinated, A- $\delta$ myelinated) are blocked first, with the larger touch and pressure fibres (A- $\Upsilon$, A- $\beta$ ) next and with the large muscle tone and postural A- $\alpha$ fibres last. It is thought that the prolonged action potential of smaller fibres provides more time for LA entry and more frequently stimulated nerves show increased 
susceptibility from a high degree of open channels.

The story does not end there, however, as in addition to blocking sodium channels, there is evidence of binding to human cardiac potassium channels (hKv1.5) to block repolarisation of the membrane, as demonstrated with ropivacaine. ${ }^{6}$ Bupivacaine and ropivacaine have also been shown to block L-type calcium channels in rat cerebrocortical membranes. From a systemic viewpoint, LAs may improve pain by inhibiting local inflammatory response to injury by decreasing inflammatory cytokine release from neutrophils.

\section{CLINICAL PHARMACOLOGY}

Potency is decided by the lipid solubility of the agent and can be expressed as a lipid:water partition coefficient (LW), the ratio of the LA in each physical phase. High coefficients increase lipophilic properties and so allow for rapid passage across the cell membrane, thus facilitating potency. Onset of action is determined by the ionisation constant or pKa value, which determines the proportion of ionised to un-ionised form of the agent at a given $\mathrm{pH}$. Potency:toxicity ratio is a useful evaluation to consider (see Table 2). Articaine has the best ratio making it clinically efficacious as well as safe.

\section{LOCAL ANAESTHETIC SYSTEMIC TOXICITY}

\section{Incidence}

Epidemiological reports have been clinically diverse and with different outcome measures used, but overall rates of systemic toxicity have been reported in France to be $0-20$ per 10,000 in 2002 and greatly dependent on the site of peripheral nerve block. ${ }^{7}$

Dentists administer thousands of local anaesthetic injections every day with few adverse events. However, local anaesthetic systemic toxicity (LAST) can occur even with the most experienced practitioner. Human error misjudging dose, anatomy, patient factors or just bad luck can contribute to the unintended development of serious systemic complications. Lignocaine is the most common LA used in dentistry and has been reported to cause systemic toxicity. ${ }^{8,9}$ Articaine, even with its excellent safety profile, may cause

Table 1 Pharmacology of common local anaesthetics

\begin{tabular}{l|l|l|l|l|l|l|l}
\hline Anaesthetic & Potency & Pot:Tox & LWPC & Onset & pKa & T11/2 $1 / 2(\mathrm{~min})$ & $\%$ PB \\
\hline Bupivacaine & 8 & 2 & 27.5 & Slow & 8.1 & 162 & 95.6 \\
\hline Articaine & 3 & 3.3 & 17 & Fast & 7.8 & 20 & 94 \\
\hline Lignocaine & 2 & 2 & 2.9 & Fast & 7.9 & 96 & 64.3 \\
\hline Mepivacaine & 2 & 2.2 & 19.3 & Fast & 7.8 & 114 & 78 \\
\hline Prilocaine & 2 & 2.7 & 0.9 & Fast & 7.7 & 93 & 55 \\
\hline Ropivacaine & 4 & 2.25 & 2.9 & Mod & 8.1 & 96 & 94 \\
\hline PB = protein binding & & & & & & & \\
\hline
\end{tabular}

\section{Table 2 Risk factors involved in LA toxicity}

\begin{tabular}{l|l|l} 
Site of injection & Drug & Patient factors \\
\hline \begin{tabular}{l|l} 
Surface area \\
Vascularity
\end{tabular} & Potency & Age \\
& Dose (volume $\times$ concentration) & Genetics \\
Vasoactivity & Cardiac pathology \\
& \pm Vasoconstrictor & Drug interactions \\
& & Pregnancy \\
& & Acidosis \\
& & Hypoxia \\
& Hypercarbia
\end{tabular}

systemic intoxication if unintentional intravascular injection is performed during a block. It has been reported that the rate of intravenous injection for inferior alveolar nerve block is as high as $15.3 \%$, which can occur due to the high vascularisation of the oral mucosa. ${ }^{10}$

\section{Clinical manifestations}

The symptoms of LA toxicity are an extension of their pharmacological profile. Toxicity manifests as a 'biphasic' effect on both the central nervous system and cardiovascular system, the two areas highly sensitive to changes in tissue electrophysiology. As plasma LA rises beyond safe levels, initial excitation occurs in both the CNS and CVS, followed by a depression with profound consequences. Generally CNS signs appear first unless very high plasma levels of anaesthetic agent are achieved or bupivacaine is used, with peri-oral paraesthesia, dizziness, tinnitus, then later drowsiness, tonic-clonic seizures and coma occurring. The more severe cardiovascular toxicity generally ensues later with early excitation: tachycardia, ventricular arrhythmias and hypertension; and leads to cardiac depression, bradycardia, and cardiovascular collapse. Other EKG changes include prolongation of the QRS complex and PR interval. ${ }^{11}$
Of particular importance is the nature of this collapse, with high incidence of LA cardiac arrest being resistant to standard resuscitative measures.

\section{Toxic plasma levels}

Systemic toxicity from local anaesthetic overdose occurs due to inadvertent intravascular injection, absorption from tissue depot, or repeated doses without balanced elimination. The concentration of bupivacaine present in the aqueous portion of plasma is directly related to the myocardial tissue absorption, and hence cardiotoxicity. ${ }^{12}$ The degree of toxicity is therefore dependent on plasma levels of LA, with highly aerobic tissues vulnerable to hypoxia, the most vulnerable being the myocardium, lungs and central nervous system.

For regional blocks, the plasma levels of lignocaine are typically 3-5 $\mu \mathrm{g} / \mathrm{ml}$, with toxic plasma levels seen at only 6-10 $\mu \mathrm{g} / \mathrm{ml}$.

\section{Risk factors}

Intuitively, one would speculate that the plasma levels of a given dose of drug would have strong correlation to the weight or body mass index of the individual. In the case of LAs, this is largely true in children, but in adults we see that the methods of administration, nature of the drug preparation and the physiological status of 


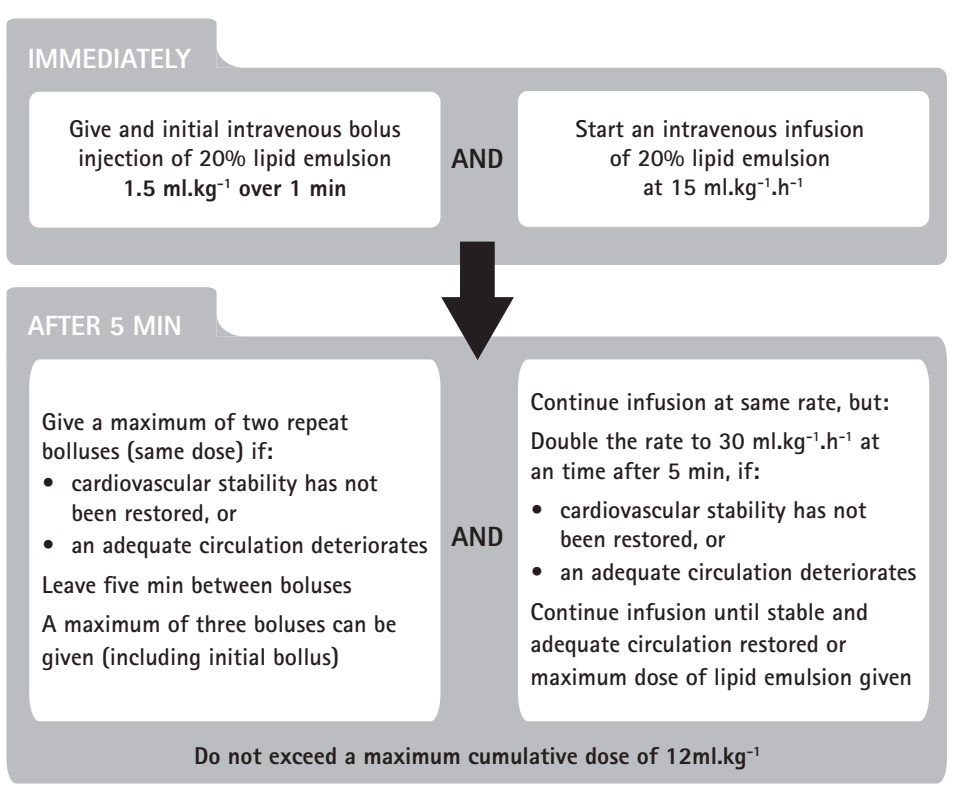

Fig. 2 AAGBI Local Anaesthetic Toxicity Guideline 2010. ${ }^{31}$ Reproduced with the kind permission of the Association of Anaesthetists of Great Britain and Ireland

the patient have far greater association. ${ }^{13}$ Underlying cardiac pathology of ischaemic heart disease, heart block and cardiac failure will render the elderly more vulnerable to toxic CVS effects. The majority of the cases of LAST seen in dentistry occur in children, as due to their small size, dose to weight ratio is more difficult to calculate and so overdose is more likely. It is also more likely to progress in adversity because a high number of blocks are performed with the child anaesthetised. The early signs of paraesthesia and mental state changes would not be detected. ${ }^{14}$ Conversely some studies show that newborns and children can actually tolerate higher plasma levels of bupivacaine compared to adults. ${ }^{15,16}$

Choice of agent also has clear implications for toxicity. Bupivacaine is more cardiotoxic than shorter-acting lignocaine, with smaller doses often resulting in cardiotoxic symptoms without prior CNS effects. ${ }^{17}$ Addition of vasoconstrictors such as adrenaline can dramatically slow the absorption of LAs from the site of injection, improving their safety and prolonging the anaesthesia, which is why higher doses of some agents are possible with a vasoconstrictor additive. Table 2 summarises the risk factors for development of LAST.

\section{Intracellular mechanisms for LAST}

In animal studies on the myocardium, lignocaine induces dramatic haemodynamic depression while bupivacaine markedly impairs both electrophysiologic and haemodynamic variables. ${ }^{18}$ In addition, in vivo and in vitro cardiac studies demonstrate bupivacaine and ropivacaine's rylation at complex I in the mitochondria, ${ }^{19}$ and block the enzyme carnitine acylcarnitine transferase used for transporting acylcarnitines across the mitochondrial membrane in fatty acids during aerobic metabolism..$^{20,21}$

Secondary to these direct effects on the myocardium, a significant cause of hypotension is due to peripheral vasodilatation from direct action on the vasculature. ${ }^{20}$

However, bupivacaine causes vasoconstriction at low doses, and so can exert a deleterious double blow on the cardiac output via negative inotropic effects and increasing afterload, which appears to be mediated by $\alpha 1$ adrenoceptors. ${ }^{22}$ Ropivicaine and levobupivacaine are far less toxic in this sense.

It should also be noted that a mechanism of toxicity appears to be inhibition of autonomic reflexes.

\section{LIPID EMULSION THERAPY}

Intralipid $20 \%$ is the first safe intravenous lipid emulsion (ILE) used in medicine and has been around since 1962 for its use in parenteral nutrition. It consists of soybean oil, egg phospholipids and glycerin and is a source of omega- 3 and -6 essential ability to uncouple oxidative phospho- fatty acids. Its use in LAST came about from an unexpected finding by Weinberg in 1998. Following a case report of a carnitine-deficient patient showing increased susceptibility to bupivacaine cardiotoxicity, he postulated the impaired fatty acid oxidation was to blame and in seminal work, pre-loaded rats with ILE before bupivacaine in hope to establish this. The result was quite the opposite, with an increase in the mean lethal dose (LD50) by $50 \% .{ }^{23}$ He later went further to demonstrate the efficacy of ILE by rescuing dogs from bupivacaine-induced cardiac arrest. ${ }^{24}$

ILE therapy for treatment of LAST is now well-established, following a crop of over 19 peer-reviewed case reports appearing since Rosenblatt's successful application of ILE to clinical practice in 2006, ${ }^{25}$ and supports the use of ILE for bupivacaine, levobupivacaine, and ropivacaine cardiotoxicity. ${ }^{14,25-29}$ This year we also see a successful case report from seemingly intractable lignocaine-induced cardiac arrest. $^{30}$ This evidence strongly supports the use of ILE in the resuscitation of LAST and because of this efficacy, ILE is has been incorporated into safety guidelines for management of LA-induced cardiotoxicity in the UK since 2007 and in the US since 2008. ${ }^{31,32}$ In 2010, the American Society of Regional Anesthesia and Pain Medicine published its practice advisory on LAST, ${ }^{33}$ highlighting the importance of airway management and early cardiopulmonary resuscitation with addition of ILE therapy.

\section{Mechanism}

ILE forms a 'lipid sink', an expanded intravascular lipid phase that acts to absorb the offending circulating lipophilic toxin, hence reducing the unbound free toxin available to bind to the myocardium. ILE therapy has shown clear superiority over adrenaline and/or vasopressin in rats that is directly linked to reduced myocardial tissue content and improved cardiac function. ${ }^{12}$

In addition, ILE:

1. Helps washout of bupivacaine ${ }^{34}$

2. Acts as a fatty acid energy substrate to the myocardium, countering the deleterious effect of LAs on fatty acid delivery to the mitochondria. ${ }^{35,36}$ 


\section{Regimen}

Initial case reports showed ILE to often succeed after standard resuscitation had failed and led to the suggestion of ILE as a 'last resort' in severe resuscitation resistant LAST. However, there is growing evidence to support its use early in the management with successful case reports supporting the immediate use in cardiac arrest (see Fig. 2). ${ }^{37-40}$

Development of optimal dosing regimens for different patient groups is on the horizon and this year ILE has been recommended for use in obstetrics. ${ }^{41}$ Support for ILE in paediatric LAST can be seen from a recent case report of ropivacaine and lignocaine-induced toxicity in a 13-yearold girl after lumbar plexus block. ${ }^{26}$ Ventricular tachycardia was impressively converted to sinus rhythm after a bolus of $3 \mathrm{ml} / \mathrm{kg}$ of lipid emulsion was given over 3 minutes. This is encouraging to read and also poses the question as to whether we need to develop optimal dosing regimens for children.

There exists debate about the use of vasopressors with ILE for treatment of LAST, and what combination, if any, is beneficial. ${ }^{42}$ At present it is not advised to deviate from standard cardiac resuscitation guidelines, with the addition of ILE therapy.

\section{PREVENTION OF TOXICITY}

The rate of intravenous injection for inferior alveolar nerve block is still as high as $15.3 \% .{ }^{10}$ The following methods, although singularly unproven, likely promote safety:

- Incremental injection of 3-5 ml aliquots with pause of one circulation time between each, although increases risk of needle migration

- Aspirate needle before each injection (but 2\% false negatives).

To this end, it is important not to exceed the safe dose of local anesthetic involved, ${ }^{43}$ which can be calculated as dose $=$ the product of volume $\times$ concentration, so that $1 \mathrm{ml}$ of lignocaine $1 \%=10 \mathrm{mg}$. The cardiotoxic potential of the amide local anaesthetics can be expressed as a maximum safe dose for administration. Table 3 highlights the safe doses of common LAs.

The relatively high toxicity of bupivacaine led it to be the main agent implicated in toxicity research. Ropivacaine

\begin{tabular}{l|l|}
\hline \multicolumn{2}{|l|}{ Table 3 Safe doses of common LAs } \\
\hline LA & $\begin{array}{l}\text { Maximum safe dose } \\
(\mathrm{mg} / \mathrm{kg})\end{array}$ \\
\hline Bupivacaine & 2.0 \\
\hline Levobupivacaine & $2.5-3.0$ \\
\hline Articaine & 7.0 \\
\hline Lignocaine & 4.0 \\
with ephinephrine & 7.0 \\
\hline Mepivacaine & 7.0 \\
\hline Prilocaine & 6.0 \\
\hline Ropivacaine & $3.0-4.0$ \\
\hline
\end{tabular}

and levobupivacaine are S-enantiomer pipecoloxylidines that have improved safety profiles compared to racemic bupivacaine. A recent study by Tsuchiya et al. investigating the interaction of racemic bupivacaine and R+ and S- enantiomers of bupivacaine and ropivacaine with biomimetic membranes of chiral lipids, demonstrated the greater interaction of the $\mathrm{R}+$ enantiomers, with S- ropivacaine presenting least influence of all. This is consistent with reported clinical cardiotoxicity of the agents and also supports the hypothesis of potency increasing the lipid bilayer membrane fluidity. ${ }^{5}$ For regional blocks involving sites of high vascularity, the use of alternative long acting amide levoenantiomers may be vindicated to further reduce the risk to patients, and this has already been suggested in dentistry for interior alveolar nerve blocks. ${ }^{44}$ However, a median effective dose study shows ropivacaine and levobupivacaine to respectively have 35\% and 3\% reduced analgesic potency to racemic bupivacaine, and so decisions to use these safer agents must be balanced against a loss of clinical efficacy. ${ }^{45}$

\section{SURGEONS' AWARENESS}

Where local anaesthesia is provided by non-anaesthetists, misdiagnosis and under-reporting of LA-associated complications is likely. ${ }^{46}$ This includes offices, outpatients and small surgical centres, and so the true incidence of LAST in these settings is unclear. There are, however, case reports of significant morbidity following LA use in such areas. ${ }^{47-50}$ The importance of surgeons' knowledge of safe use of LAs and management of complications is signified by the reported incidence of five deaths from suspected lignocaine systemic toxicity or related complications following tumescent liposuction in New York between $1993-1998 . .^{51-54}$ It is of interest to note that this procedure is still very popular today and commonly performed without the presence of an anaesthetist. Also of concern is a recent survey in the UK by Collins that suggests only half of hospital surgeons know how to calculate the correct dose of local anaesthetic being used and fewer than 25\% of non-anaesthetic doctors knew the recommended safe doses. Only $7 \%$ of non-anaesthetic doctors knew the correct treatment to be intralipid and only 3\% knew the initial dose. ${ }^{55}$ These findings highlight the importance of education, which is of particular significance to practitioners who regularly use LAs without the presence of an anaesthetist.

\section{SUMMARY}

Vigilance is required when performing procedures that have a potential for systemic toxicity. There are numerous examples of local anaesthetic systemic complications in the literature, many in the hands of non-anaesthetists. We see that strategies to reduce the risk of LAST can never eliminate its risk. Although uncommon, the consequences can be fatal. Advances in ILE therapy and understanding is providing life-saving rescue in the most dreaded situations faced by practitioners, and further progress will likely improve our safe use of LAs in the future. Rapid identification of toxicity and a good recall of the ILE therapy regimen can save lives but we need to expand awareness to practitioners in remote locations such as outpatients, offices and especially those who work without an anaesthetist. We encourage these facilities to put together a 'rescue kit' in a specified location with the current guidelines readily available. LAs are used more frequently by surgeons and dentists than anaesthetists, and on that note we feel that the respective colleges should also develop guidelines for management of LAST incorporating lipid emulsion therapy. abuse of cocaine: suggested by its invariably successful employment in more than a thousand minor surgical operations. NY Med J 1885; 42: 294-295.

2. Nizharadze N, Mamaladze M, Chipashvili N, Vadachkoria D. Articaine - the best choice of local 
anesthetic in contemporary dentistry. Georgian Med News 2011; 190: 15-23.

3. Ritchie J M, B. Ritchie B, Greengard P. The active structure of local anesthetics. J Pharmacol Exp The 1965; 150: 152-159

4. Tsuchiya H, Ueno T, Mizogami M, Takakura K. Local anesthetics structure-dependently interact with anionic phospholipid membranes to modify the fluidity. Chem Biol Interact 2010; 183: 19-24.

5. Tsuchiya $H$, Ueno T, Mizogami M. Stereostructurebased differences in the interactions of cardiotoxic local anesthetics with cholesterol-containing biomimetic membranes. Bioorg Med Chem 2011; 19: $3410-3415$

6. Valenzuela C, Delpón E, Franqueza L, Gay P, Snyders $D$ J, Dunwiddie TV. Effects of ropivacaine on a potassium channel (hKv1.5) cloned from human ventricle. Anesthesiology 1997; 86: 718-728.

7. Auroy $Y$, Benhamou D, Barques L et al. Major complications of regional anaesthesia in France: the SOS Regional Anesthesia Hotline Service. Anesthesiology 2002; 97: 1274-1280.

8. Mehra P, Caiazzo A, Maloney P. Lidocaine toxicity. Anesth Prog 1998; 45: 38-41.

9. Virts B E. Local anesthesia toxicity review. Pediatr Dent 1999; 21: 375.

10. Zenouz A, Ebrahimi H, Mahdipour M, Pourshahidi S, Amini $P$, Vatankhah $M$. The incidence of intravascular needle entrance during inferior alveolar nerve block injection. J Dent Res Dent Clin Dent Prospects 2008 2: 38-41

11. Heavner J E. Cardiac toxicity of local anaesthetics in the intact isolated heart model: a review. Reg Anesth Pain Med 2002; 27: 545-555.

12. Weinberg G, Lin $B$, Zheng $S$ et al. Partitioning effect in lipid resuscitation: further evidence for the lipid sink. Crit Care Med 2010; 38: 2268-2269.

13. Rosen M A, Jone R M, Yano Y, Budinger T F. Bupivacaine-induced cardiotoxicity in hypoxic and acidotic sheep. Anesth Analg 1985; 64: 1089-1096.

14. Foxall G, McCahon R, Lamb J, Hardman J G, Bedforth N M. Levobupivacaine-induced seizures and cardiovascular collapse treated with Intralipid. Anaesthesia 2007; 62: 516-518.

15. Berde C B. Toxicity of local anesthetics in infants and children. J Pediatr 1993. 122:14-20.

16. De Negri P, Ivani G, Tirri T, Del Piano A C. New local anaesthetics for pediatric anaesthesia. Curr Opin Anaesthesiol 2005; 18: 289-292.

17. Albright $\mathrm{G} A$. Cardiac arrest following regional anesthesia with etidocaine or bupivacaine. Anaesthesiology 1979; 51: 285-287.

18. Bruelle P, LeFrant J Y, de La Coussaye J E et al. Comparative electrophysiologic and hemodynamic effects of several amide local anesthetic drugs in anesthetised dogs. Anesth Analg 1996; 82: 648-656.

19. Maughan D W. Kinetics and energetics of the crossbridge cycle. Heart Fail Rev 2005; 10: 175-185.

20. Newton D J, McLeod G A, Khan F, Belch J J. Vasoactive characteristics of bupivacaine and levobupivacaine with and without adjuvant adrenaline in peripheral human skin. Br J Anaesth 2005; 94: 662-667.

21. Weinberg $G L$, Palmer J W, VadeBoncouer $T R$, Zuechner M B, Edelman G, Hoppel C L. Bupivacaine inhibits acylcarnitine exchange in cardiac mitochondria. Anesthesiology 2000; 92: 523-528.

22. Royse $C$ F, Royse A G. The myocardial and vascular effects of bupivacaine, levobupivacaine, and ropivacaine using pressure volume loops. Anesth Analg 2005; 101: 679-687.

23. Weinberg $G L$, VadeBoncouer $T R$, Ramaraju $G A$, Garcia-Amaro M F, Cwik M J. Pretreatment or resuscitation with a lipid infusion shifts the doseresponse to bupivacaine-induced asystole in rats. Anesthesiology 1998; 88: 1071-1075.

24. Weinberg G L, Ripper R, Feinstein D L, Hoffman $W$. Lipid emulsion infusion rescues dogs from bupivacaine-induced cardiac toxicity. Reg Anesth Pain Med 2003; 28: 198-202.

25. Rosenblatt M A, Abel M, Fischer G W, Itzkovich C J, Eisenkraft J B. Successful use of a 20\% lipid emulsion to resuscitate a patient after a presumed bupivacaine-related cardiac arrest. Anesthesiology 2006; 105: 217-218.

26. Ludot $H$, Tharin J Y, Belouadah M, Marzoit J $X$ Malinovsky J M. Successful resuscitation after ropivacaine and lidocaine-induced ventricular arrhythmia following posterior lumbar plexus block in a child. Anesth Analg 2008; 106: 1572-1574.

27. Litz R J, Roessel T, Heller A R, Stehr S N. Reversal of central nervous system and cardiac toxicity after local anesthetic intoxication by lipid emulsion injection. Anesth Analg 2008; 106: 1575-1577.

28. Warren J A, Thoma R B, Georgescu A, Shaj S J. Intravenous lipid infusion in the successful resuscitation of local anesthetic-induced cardiovascular collapse after supraclavicular brachial plexus block. Anesth Analg 2008; 106: 1578-1580.

29. Cave G., Harvey M, Graudins A. Intravenous lipid emulsion as antidote: a summary of published human experience. Emerg Med Australas 2011; 23: 123-141.

30. Dix S K, Rosner G F, Nayar M, et al. Intractable cardiac arrest due to lidocaine toxicity successfully resuscitated with lipid emulsion. Crit Care Med 2011; 39: 872-874.

31. AAGBI. Management of severe local anaesthetic toxicity. AAGBI Safety Guideline 2010; online article available at http://www.aagbi.org/sites/default/files/ la toxicity 2010_0.pdf

32. Gabrielli A, O'Connor M F, Maccioli G A. Anesthesia advanced circulatory life support. The American Society of Critical Care Anesthesiologists and The American Society of Anesthesiologists, Committee on Critical Care Medicine, 2008.

33. Neal J M, Bernards C M, Butterworth J F 4th et al. ASRA practice advisory on local anesthetic systemic toxicity. Reg Anesth Pain Med 2010; 35: 152-161.

34. Weinberg G L, Ripper R, Murphy P et al. Lipid infusion accelerates removal of bupivacaine and recovery from bupivacaine toxicity in the isolated rat heart. Reg Anesth Pain Med 2006; 31: 296-303.

35. Silveira L, Hirabara H M, Alberici L C et al. Effect of lipid infusion on metabolism and force of rat skeletal muscles during intense contractions. Cell Physiol Biochem 2007; 20: 213-26.

36. Coat M, Pennec J P, Guillouet M, Arvieux C C, Gueret G. [Haemodynamic effects of intralipid after local anaesthetics intoxication may be due to a direct effect of fatty acids on myocardial voltage-dependent calcium channels]. Ann Fr Anesth Reanim 2010; 29: 661.
37. Weinberg $G \mathrm{~L}$. Intravenous lipid emulsion: why wait to save a life? Emerg Med Australas 2011; 23: 113-115.

38. Markowitz S, Neal J M. Immediate lipid emulsion therapy in the successful treatment of bupivacaine systemic toxicity. Reg Anesth Pain Med 2009; 34: 276.

39. Charbonneau H, Marcou T A, Mazoit J X, Zetlaoui P J, Benhamou D. Early use of lipid emulsion to treat incipient mepivacaine intoxication. Reg Anesth Pain Med 2009; 34: 277-278.

40. Sonsino D H., Fischler M. Immediate intravenous lipid infusion in the successful resuscitation of ropivacaine-induced cardiac arrest after infraclavicular brachial plexus block. Reg Anesth Pain Med 2009; 34: 276-277.

41. Bern S, Weinberg G. Local anaesthetic toxicity and lipid resuscitation in pregnancy. Curr Opin Anaesthesio/ 2011; 24: 262-267.

42. Hiller D B, Gregoria G D, Ripper R et al. Epinephrine impairs lipid resuscitation from bupivacaine overdose: a threshold effect. Anesthesiology 2009; 111: 498-505.

43. Rosenberg P H, Veering B T, Urmey W F. Maximum recommended doses of local anesthetics: a multifactorial concept. Reg Anesth Pain Med 2004; 29: 564-575

44. Branco F P, Ranali J, Ambrosano G M, Volpato M C. A double-blind comparison of $0.5 \%$ bupivacaine with 1:200,000 epinephrine and 0.5\% levobupivacaine with $1: 200,000$ adrenaline for the inferior alveolar nerve block. Oral Surg Oral Med Oral Patho Oral Radiol Endod 2006; 101: 442-447.

45. Lee Y Y, Kee W D N, Fong S Y, Liu J T C, Gin T. The median effective dose of bupivacaine, levobupivacaine, and ropivacaine after intrathecal injection in lower limb surgery. Anesth Analg 2009; 109: 1331-1334.

46. Weinberg G L. Lipid Rescue. Available from: http://www.lipidrescue.org/.

47. Dorf E, Kuntz A F, Kelsey J, Holstege C P. Lidocaineinduced altered mental status and seizure after haematoma block. J Emerg Med 2006; 31: 251-253.

48. Marra, D E, Yip D, Fincher E F, Moy R L. Systemic toxicity from topically applied lidocaine in conjunction with fractional photothermolysis. Arch Dermato/ 2006; 142: 1024-1026.

49. Donald M J, Derbyshire S. Lignocaine toxicity; a complication of local anaesthesia administered in the community. Emerg Med J 2004; 21: 249-250.

50. Smith M., Wolfram W, Rose R. Toxicity - seizures in an infant caused by (or related to) oral viscous lidocaine use. J Emerg Med 1992; 10: 587-590.

51. Martinez M A, Ballesteros S, Segura L J, Garcia M. Reporting a fatality during tumescent liposuction. Forensic Sci Int 2008; 178: e11-e16.

52. Klein J A, Kassarjdian N. Lidocaine toxicity with tumescent liposuction. A case report of probable drug interactions. Dermatol Surg 1997 23: $1169-1174$.

53. Seigne R. Lignocaine toxicity--a surgical surprise Anaesthesia 1997; 52: 91

54. Rao R B, Ely S F, Hoffman R S. Deaths related to liposuction. N Engl J Med 1999; 340: 1471-1475.

55. Collins J. Awareness of local anaesthetic toxicity issues among hospital staff. Anaesthesia 2010; 65: 960-961. 